Original Research Paper

\title{
Poly(3,4-Ethylenedioxythiophene):Poly(4-Styrenesulfonate) Anode for ITO-Free Organic Light-Emitting Diodes
}

\author{
${ }^{1}$ Keanchuan Lee and ${ }^{2}$ Martin Weis \\ ${ }^{I}$ Department of Fundamental and Applied Sciences, \\ Universiti Teknologi Petronas, 32610 Seri Iskandar, Perak, Malaysia \\ ${ }^{2}$ Institute of Electronics and Photonics, \\ Slovak University of Technology, Ilkovicova 3, 81219 Bratislava, Slovakia
}

\author{
Article history \\ Received: 23-11-2017 \\ Revised: 05-12-2017 \\ Accepted: 20-12-2017 \\ Corresponding Author: \\ Keanchuan Lee \\ Department of Fundamental \\ and Applied Sciences, \\ Universiti Teknologi Petronas, \\ 32610 Seri Iskandar, Perak, \\ Malaysia \\ Email: lee.kc@utp.edu.my
}

\begin{abstract}
Organic Light-Emitting Diodes (OLEDs) devices using flexible substrates are envisioned for flexible, low-cost and roll-to-roll mass production. However, transparent conductive oxides such as indium tin oxide (ITO) do not fit the requirements of flexible devices, fabricated without vacuum-based technology. Hence, conductive polymer poly $(3,4-$ ethylenedioxythiophene):poly(4-styrenesulfonate) (PEDOT:PSS) has been proposed as an alternative approach to transparent conductive electrodes. The secondary doping of PEDOT:PSS shows improvement of conductivity to reasonable level and due to lower injection barrier devices with polymer anode exhibit even better performance.
\end{abstract}

Keywords: Flexible, OLED, PEDOT:PSS, Anode

\section{Introduction}

Organic electronics have gained tremendous research interest for perspective application in optoelectronic devices such as Organic Light-Emitting Diodes (OLEDs), or organic solar cells since it has been envisioned for flexible, low-cost and roll-to-roll mass production (Katz, 2004; Sirringhaus, 2005). All these devices are usually represented by the sandwich structures and require application of transparent conductive layer for one of electrodes. However, 'transparent conductors' are neither optically transparent nor metallically conductive. The combination of these two properties in one material contradict each other. As a result, the transparent conductors are all about the balance in between the transparency in visible wavelengths and sufficient conductivity (Facchetti and Marks, 2010).

Chiang et al. (1977) in few decades ago suggested $\pi$ conjugated polymers as a promising candidate for organic conductors and as a possible alternative to transparent conductive oxides. Even though various conductive polymers such as polyacetylene, polypyrrole, polythiophene, or polyphenylhave been investigated (Malhotra, 2002), the most popular material is still Poly(3,4-Ethylenedioxythiophene) (PEDOT) because of high conductivity. On the other hand, low solubility of PEDOT caused difficulty in thin layer fabrication and the mixture of PEDOT with
Poly(4-Styrenesulfonate) (PSS) have been proposed to improve solubility in aqueous solutions. Polymer mixture poly(3,4-ethylenedioxythiophene):poly(4styrenesulfonate)(PEDOT:PSS) has been patented already in 1988 by Bayer AG (Jonas et al., 1988); however, commercial applications are not common until today. One of the most significant limitations was insufficient electrical conductivity ranging from $10^{-6}$ to $10^{-3} \mathrm{~S} / \mathrm{cm}$ (Nardes et al., 2007). The work function of about $5.0 \mathrm{eV}$ (Nardes et al., 2008) makes the polymer applicable as the hole injection layer even though the conductivity is not high. In details, the ITO anode surface has been modified by the PEDOT:PSS thin film to enhance the charge injection andincrease the overall device performance ( $\mathrm{Mu}$ et al., 2007 \& Hong et. Al., 2008). The need of high conductivity for deice applications inspired many research groups to improve the conductivity by polymer doping. It has been reported that various polyols, amides, sulfoxides, anionic surfactant and salts have a capability to increase the conductivity by 2 or 3 orders of magnitude (Elschner et al., 2010). All these additives are usually denoted as "secondary dopants" (MacDiarmid and Epstein, 1994), since conductive polymer PEDOT is already doped by PSS as a counter ion to create a polyelectrolyte complex. It should be mentioned here that secondary dopants are not present in fabricated PEDOT:PSS films and that they act as co-solvents only (Nevrela et al., 2015). 
In present work we report the properties of OLED device with a PEDOT:PSS anode as an alternative to ITO. Significantly higher current density and electroluminescence light intensity were achieved using the PEDOT:PSS anode what illustrates superior charge injection in comparison with conventional ITO anode. As a result, although the PEDOT:PSS layer has still higher sheet resistance than ITO layer, the overall device performance achieve higher level and it demonstrates ITO-free alternative for OLED fabrication technology.

\section{Experiment}

Transparent glass has been used as substrate for all OLED devices. Indium tin oxide (ITO) anode with resistivity of about $10 \mathrm{Ohm} / \mathrm{sq}$ was patterned using hydrochloric acid. The poly(3,4ethylenedioxythiophene):poly(4-styrenesulfonate)

(PEDOT:PSS) aqueous solution (Clevios PH 1000, 1.1\% solid content in water with PEDOT:PSS ratio 1:2.5) was supplied by Hereaus, Germany. For PEDOT:PSS doping, the secondary doping method by sorbitol (Sigma-Aldrich) was employed to increase the conductivity on the level of $1000 \mathrm{~S} / \mathrm{cm}$ (Nevrela et al., 2012). The concentration of secondary dopant in solution was $5 \mathrm{wt} \%$. Prior deposition was solution filtered through 0.25 micrometer syringe filters to remove insoluble particles. Solution was spun using $3000 \mathrm{rpm}$ to obtain $100 \mathrm{~nm}$ thick layer. PEDOT:PSS layer was subsequently heated on hotplate at the temperature of $120^{\circ} \mathrm{C}$ to remove residual solvent. Hole transport layer of N,N'-di-1-naphthyl-N,N'-diphenyl-1,10-biphenyl-4,40diamine ( $\alpha-\mathrm{NPD}$, Sigma-Aldrich) and emissive/electron transport layer of tris(8-quinolinolato) aluminum (Alq3, Sigma-Aldrich) were evaporated in vacuum better than $10^{-}$ ${ }^{5} \mathrm{~Pa}$ to obtain thicknesses of 150 and $50 \mathrm{~nm}$, respectively. The evaporation rate was kept constant on $3 \mathrm{~nm} / \mathrm{min}$. The aluminum ( $\mathrm{Al}$ ) cathode was subsequently evaporated through the shadow mask. Figure 1 depicts schematic view of fabricated OLED devices.

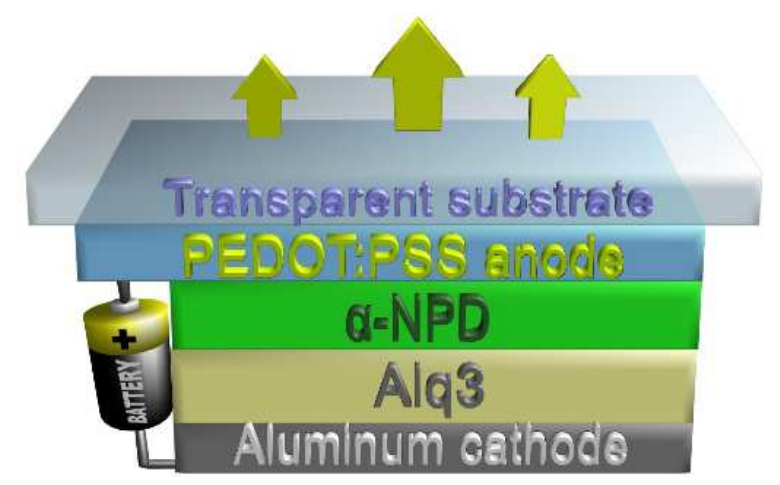

Fig. 1: Schematic view of fabricated OLED device with PEDOT:PSS anode

\section{Results and Discussion}

Figure 2 depicts the typical current-voltage and electroluminescence properties of OLEDdevices using ITO or PEDOT:PSS layers as anodes. The device with PEDOT:PSS anode has obviously better performance in comparison with ITO-based device. After a threshold voltage of about $3 \mathrm{~V}$ the device with PEDOT:PSS anode always exhibits current of one magnitude higher than the device with ITO anode. Note that both devices have been fabricated simultaneously; hence, the organic semiconductors are identical in the meaning of thicknesses and/or defect concentrations.

Furthermore, the current dependence of luminance efficiency, shown in Fig. 3, illustrates device improvement in broad range of applied currents. In other words, not only the output currents are achieved higher, but also the overall device efficiency. This result depicts that the balance of electron-hole densities is more favorable for device with PEDOT:PSS anode.

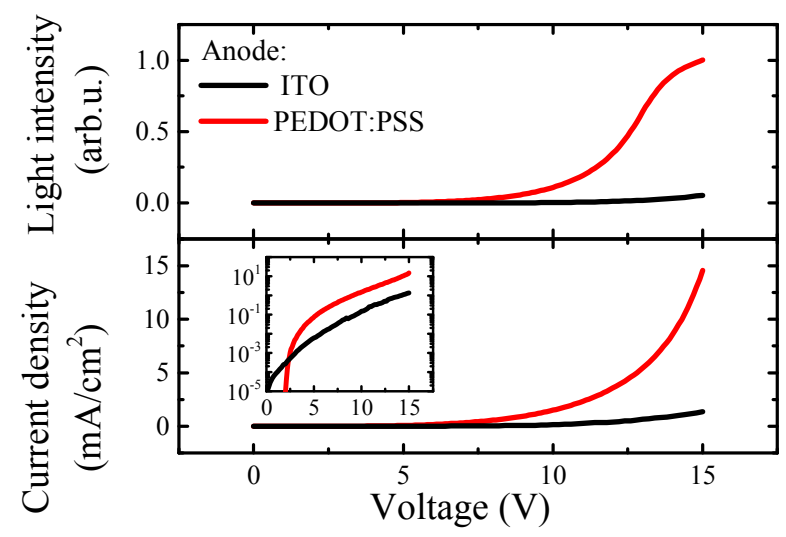

Fig. 2: Voltage dependencies of current density and electroluminescence light intensity of OLED devices using ITO or PEDOT:PSS anodes

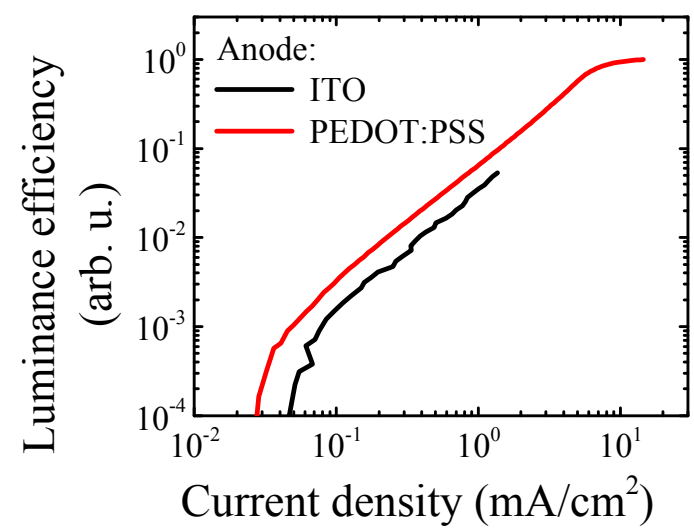

Fig. 3: The current dependence of luminance efficiency of OLED devices using ITO or PEDOT:PSS anodes. 


\section{Conclusion}

Recent progress in the field of nanostructured and organic materials found conductive polymers as promising candidates for transparent conductive oxide replacement. The application of organic conductors for transparent electrode in OLED device has specific requirements on optical transparency, electrical conductivity, charge injection properties, surface morphology, as well as environmental stability. Even though the secondary doping of PEDOT:PSS was applied to improve the conductivity, the sheet resistance is still one order of magnitude higher than one of ITO layer. On the other hand, the overall OLED device performance with PEDOT:PSS anode is higher due to smooth charge injection. It demonstrates that conductive polymers, namely PEDOT:PSS, provide sufficient sheet resistance, high optical transparency in visible region and low-cost "wet" fabrication technology what make them suitable for future flexible OLED applications.

\section{Acknowledgement}

This work was supported by Fundamental Research Grant Scheme (FRGS) with cost-center number 0153AB-I97.

\section{Author's Contributions}

Both authors equally contributed in this work.

\section{Ethics}

The authors declare no competing financial interest.

\section{References}

Chiang, C.K., C.R. Fincher Jr., Y.W. Park, A.J. Heeger and H. Shirakawa et al., 1977. Electrical conductivity in doped polyacetylene. Phys. Rev. Lett., 39: 1098-1098.

DOI: 10.1103/PhysRevLett.39.1098

Elschner, A., S. Kirchmeyer, W. Lövenich, U. Merker and K. Reuter, 2010. PEDOT: Principles and Applications of an Intrinsically Conductive Polymer. 1st Edn., CRC Press, Boca Raton, ISBN-10: 1420069128, pp: 377.
Facchetti, A. and T.J. Marks, 2010. Transparent Electronics: From Synthesis to Applications. 1st Edn., Wiley, Chichester, ISBN-10: 0470710594, pp: 470.

Hong, W., Xu, Y., Lu, G., Li, C. and Shi, G., 2008. Transparent graphene/PEDOT-PSS composite films as counter electrodes of dye-sensitized solar cells. Electrochem. Commun., 10(10): 1555-1558. DOI: $10.1016 /$ j.elecom.2008.08.007

Jonas, F., G. Heywang and W. Schmidtberg, 1988. German patent DE 3813589 A1. Prior, (Bayer AG), Bayer AG.

Katz, H.E., 2004. Recent advances in semiconductor performance and printing processes for organic transistor-based electronics. Chem. Mater., 16: 4748-4756. DOI: $10.1021 / \mathrm{cm} 049781 \mathrm{j}$

MacDiarmid, A.G. and A.J. Epstein, 1994. The concept of secondary doping as applied to polyaniline. Synth. Metals, 65: 103-116. DOI: 10.1016/0379-6779(94)90171-6

Malhotra, B.D., 2002. Handbook of Polymers in Electronics. 1st Edn., iSmithers Rapra Publishing, Shropshire, ISBN-10: 1859574386, pp: 488.

$\mathrm{Mu}, \mathrm{H} ., \mathrm{W}$. Li, R. Jones, A. Steckl and D. Klotzkin, 2007. A comparative study of electrode effects on the electrical and luminescent characteristics of $\mathrm{Alq}_{3} / \mathrm{TPD}$ OLED: Improvements due to conductive polymer (PEDOT) anode. J. Lumin., 126: 225-229. DOI: 10.1016/j.jlumin.2006.07.005

Nardes, A.M., M. Kemerink and R.A.J. Janssen, 2007. Anisotropic hopping conduction in spin-coated PEDOT:PSS thin films. Phys. Rev. B, 76: 085208085208. DOI: 10.1103/PhysRevB.76.085208

Nardes, A.M., M. Kemerink, M.M. de Kok, E. Vinken and K. Maturova et al., 2008. Conductivity, work function and environmental stability of PEDOT:PSS thin films treated with sorbitol. Org. Electron., 9: 727-734. DOI: 10.1016/j.orgel.2008.05.006

Nevrela, J., M. Micjan, M. Novota, S. Kovacova and M. Pavuk et al., 2015. Secondary doping in poly(3,4ethylenedioxythiophene):Poly(4-styrenesulfonate) thin films. J. Pol. Sci. Pol. Phys., 53: 1139-1146. DOI: $10.1002 /$ polb.23754

Sirringhaus, H., 2005. Device physics of solutionprocessed organic field-effect transistors. Adv. Mater., 17: 2411-2425.

DOI: $10.1002 / \mathrm{adma} .200501152$ 